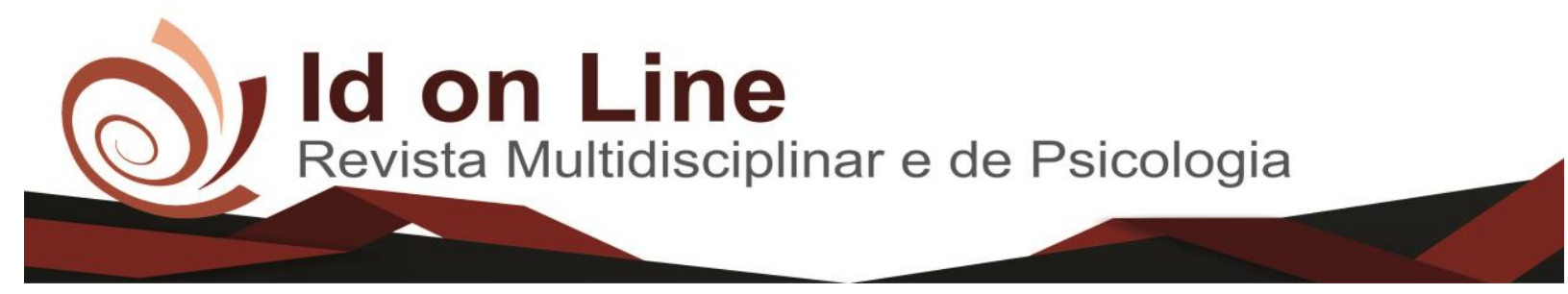

Artigo

\title{
Fatores Associados a Quedas em Idosos de uma Unidade Básica de Saúde
}

\author{
Gabriela Fernandes Lemos ${ }^{1}$, Juliana Barros Ferreira ${ }^{2}$, Kleyton Trindade Santos ${ }^{3}$,
} Luciana Araújo dos Reis ${ }^{4}$, Karla Cavalcante Silva de Morais ${ }^{5}$

\begin{abstract}
Resumo: Quedas definem-se como contato em superfície de apoio resultante mudança em nível inferior à sua posição inicial. O estudo teve por objetivo geral: identificar os fatores de risco associados a quedas em idosos de uma Unidade Básica de Saúde. Utilizou-se metodologia analítica, exploratória e quantitativa com 50 idosos que obtiveram estado cognitivo preservado que apresentaram risco de quedas. Foi aplicado questionários sociodemográficos: Mini Exame do Estado Mental, Índice de Barthel, Índice de Lawton, Avaliação Antropométrica, Time up and Go, Escalas de Depressão e Risco de Quedas. Destacou-se o fator quedas nessa população de idosos no valor (p-valor) de $<(0,001)$, onde as causas mais relevantes foram: as associadas a quedas anteriores p-valor $(0,004)$, uso de medicações p-valor de $(0,013)$ e déficit sensório com p-valor de $(0,016)$. Estes resultados contribuem estrategicamente para educação em saúde com foco em atividades preventivas, educacionais e reabilitação a minimizar surgimento de quedas.
\end{abstract}

Descritores: Acidentes por Quedas; Idosos; Prevenção; Unidade de Saúde.

\section{Factors Associated to Falls in the Elderly in a Basic Health Unit}

\begin{abstract}
Falls are defined as contact on the support surface resulting from shifting to a lower level than its initial position. The objective of the study was to identify the risk factors associated with falls in the elderly of a Basic Health Unit. An analytical, exploratory and quantitative methodology was used with 50 elderly individuals who obtained a preserved cognitive status that presented a risk of falls. Socio-demographic questionnaires were applied: Mini Mental State Examination, Barthel Index, Lawton Index, Anthropometric Assessment, Time Up and Go, Depression Scales and Falls Risk. It was highlighted the fall factor in this population of the elderly (p-value) of $<(0.001)$, where the most relevant causes were: those associated with previous falls p-value (0.004), use of p- 0.013) and sensory deficit with pvalue of (0.016). These results contribute strategically to health education with a focus on preventive, educational and rehabilitation activities to minimize the occurrence of falls.
\end{abstract}

Keywords: Accidents by Falls; Elderly; Prevention; Health Unit.

\footnotetext{
${ }^{1}$ Discente de Fisioterapia da Faculdade Independente do Nordeste - FAINOR. E-mail: gabrielaflemos@gmail.com. Vitória da Conquista, Bahia, Brasil.

${ }^{2}$ Fisioterapeuta. Mestranda em Tecnologias em Saúde pela EBMSP/BA. Docente da Faculdade Independente do Nordeste - FAINOR e Faculdade de Tecnologia e Ciências - FTC Campus Vitória da Conquista- BA. E-mail: julibarros78@ hotmail.com.br Vitória da Conquista, Bahia, Brasil.

${ }^{3}$ Fisioterapeuta. Mestre em Ciências da Saúde pela UESB. Docente da Faculdade Independente do Nordeste - FAINOR. E-mail: kleyton_santos@ hotmail.com.br Vitória da Conquista, Bahia, Brasil.

${ }^{4}$ Fisioterapeuta. Doutora em Ciências da Saúde pela UFRN. Docente da Universidade Estadual do Sudoeste da Bahia - UESB e da Faculdade Independente do Nordeste - FAINOR. E-mail: lucianareisfainor@gmail.com.br Vitória da Conquista, Bahia, Brasil.

${ }^{5}$ Fisioterapeuta. Mestre em Saúde Pública pela ENSP/FIOCRUZ . Docente da Faculdade Independente do Nordeste - FAINOR e Maurício de Nassau Campus Vitória da Conquista-BA. E-mail: karlinhakau@ hotmail.com. Vitória da Conquista, Bahia, Brasil.

Gabriela Fernandes Lemos. Avenida Recife 861, Brasil - Vitória da Conquista-BA; CEP: 45051-040. gabrielaflemos@ gmail.com.br Tel: (77) 98824-2968.
} 


\section{Introdução}

A ocorrência de quedas é potencializada em relação ao número de casos evidenciados quanto a incidência e prevalência em que são registrados no Brasil. Conforme sua natureza multifatorial, a frequiência e os resultados recorrentes nesta relação tem-se uma das grandes síndromes geriátricas na Saúde Pública (FALSARELLA, 2012).

A morbidade e mortalidade com o avanço da idade acentuam-se gradualmente em meios a outros fatores tais como a restrição da morbilidade, fraturas, depressão, incapacidade funcional, perda da independência, autonomia, institucionalização e o declínio da qualidade de vida. As condições psicológicas, socioeconômicas podem resultar em sobrecarga também no sistema de saúde público e em sua deficiência na atenção básica, a falha eficaz no atendimento pode resultar na perda de autonomia e qualidade de vida dos idosos, afetando também familiares e cuidadores, que devem adapta-se aos cuidados especiais necessários, adaptando a rotina em função da recuperação ou adaptação do idoso pós-queda (GASPAROTTO, 2012; COIMBRA, 2012).

Cair é um risco inerente a qualquer pessoa, porém ao avanço da idade, intensidade e o agravamento podem resultar em conseqüências de maior impacto aos que necessitam de maiores cuidados e atenção. Idosos possuem características especiais que carecem de atenção primordial, e o descuido pode ocasionar acidentes em situações as quais não haveriam, ou causariam queda. Tais fatores de risco situam-se também depreendidas as questões fisiológicas do envelhecimento, psicossociais, visão, audição, aparelho vestibular, propriocepção, equilíbrio, fraqueza muscular, tato e outros (PAULA, 2015).

Santos (2017), relata sobre isso quanto ao ambiente familiar, social e a fatores fora do cotidiano social regular para a ocorrência de quedas, e que sua importância observa-se na prevenção a fatores como iluminação adequada, piso não-escorregadio, mobília e objetos, disposição dos tapetes, atenção ao banho e o uso de anteparo para assento, piso apropriado, barras de apoio em escadas, degraus, banheiro, corredores e demais locais de circulação.

Portanto, devido a população mundial estar envelhecendo em intensidade e proporções crescentes, é significativa a realização de estudos nestes parâmetros aqui estudados a identificar os fatores de risco associado a quedas em idosos, avaliando e 
identificando a prevalência a que estes indivíduos estão expostos, e assim fundamentar meios para a adoção de estudos e ações a prevenção dos riscos e agravos provendo melhor qualidade de vida. Esta pesquisa teve como objetivo geral: Identificar os fatores associados a quedas em idosos de uma Unidade Básica de Saúde. E como objetivos específicos: Verificar o perfil socioeconômico e demográfico dos idosos; Comparar entre os valores médios das características antropométricas, testes motores e demais variáveis segundo o risco de quedas.

\section{Metodologia}

Essa pesquisa foi classificada como analítica exploratória e abordagem quantitativa. Gil (2017) considera pesquisa quantitativa, aquela qual expressa as variáveis em dados numéricos, utilizando-se de recursos técnicos estatísticos em sua análise como porcentagem, média, desvio padrão e coeficiente de correlação regressiva. Mais indicada devida sua precisão e confiança para planejamento de ações coletivas, seus resultados são passives a generalização em relação as amostras retratarem fielmente a população evidenciada. Os dados foram coletados em uma Unidade Básica de Saúde, situada no município de Vitória da Conquista na região do Sudoeste da Bahia.

A população estudada consistiu em 120 idosos, identificados e cadastrados em uma Unidade Básica de Saúde pesquisada. Entretanto, a amostra representativa do estudo foi composta por 50 idosos selecionados aleatoriamente ao usarem os serviços da Unidade, e que se enquadraram aos critérios de seleção da pesquisa. Como critério de inclusão: Idosos que apresentavam risco a quedas e que apresentaram estado cognitivo preservado testado a partir da aplicação prévia do Mini Exame do Estado Mental (MEEM) (FOLSTEIN; FOLSTEIN; MCHUGH, 1975). Foram excluídos do estudo os idosos com incapacidade de compreensão das perguntas, mesmo apresentando estado cognitivo adequado de acordo com o MEEM.

Para a coleta de dados foi utilizada a aplicação de um questionário abrangente, composto por variáveis sociodemográficas e econômicas, e aplicação de escalas validadas: Mini Exame do Estado Mental (MEEM), o Índice de Barthel, o Índice de Lawton, Avaliação Antropométrica, Escala de Mobilidade e Flexibilidade (TUG), Escala de Depressão Geriátrica (GDS) e a Escala de Risco de Quedas o Falls Risk Score de Dowton. 
Os dados foram coletados, transportados e analisados na descritiva das variáveis de estudo e medidas de tendência central (média, mediana, moda) e dispersão (desvio-padrão e amplitude). Estatisticamente as proporções foram avaliadas usando testes de qui-quadrado de Pearson, e significância de $5 \%(\mathrm{p}<0,05)$. Para as análises, utilizou-se os testes estatísticos: Teste t pareado; Teste Kolmogorov-Smirnov; Teste t de Studant e Teste de Mann-Whitney.

O estudo faz parte de um projeto mãe, cujo título: Quedas e Fatores Associados em Idosos, foi aprovado pelo Comitê de Ética em pesquisa (CAAE: 62170516.40000.5578), conforme rege a resolução 466/12. Todos os idosos foram esclarecidos acerca do objetivo do projeto e somente participaram aqueles que assinaram o Termo de Consentimento Livre e Esclarecido.

\section{Resultados}

Para análise é necessário conhecer a população de idosos estudada e seus aspectos sociodemográficos. Tais dados fundamentam a interpretação das representações sociais e associações a longevidade e ao risco de quedas.

Tabela 1: Frequência e análises sociodemográficas dos idosos da amostra. Vitória da Conquista/BA, 2017.

\begin{tabular}{llcc}
\hline Variável & Classe & $\mathbf{N}$ & $\mathbf{( \% )}$ \\
\hline Idade & $<70$ & 30 & $(60,00)$ \\
& $>=70$ & 20 & $(40,00)$ \\
\hline Sexo & Masculino & 19 & $(38,00)$ \\
& Feminino & 31 & $(62,00)$ \\
\hline Estado civil & Solteiro & 9 & $(18,00)$ \\
& Casado & 25 & $(50,00)$ \\
& Divorciado & 4 & $(8,00)$ \\
& Viúvo & 12 & $(24,00)$ \\
\hline Raça & Branco & 29 & $(58,00)$ \\
& Pardo & 16 & $(32,00)$ \\
& Negro & 5 & $(10,00)$ \\
& Branco & 29 & $(58,00)$ \\
& Não branco & 21 & $(42,00)$ \\
\hline
\end{tabular}




\begin{tabular}{llcc}
\hline Profissão & Aposentado & 16 & $(32,65)$ \\
Dona de casa & 14 & $(28,57)$ \\
Professor & 2 & $(4,08)$ \\
Servente de pedreiro & 1 & $(2,04)$ \\
Lavrador & 7 & $(14,29)$ \\
Doméstica & 2 & $(4,08)$ \\
Agente comunitário & 2 & $(4,08)$ \\
Carpinteiro & 2 & $(4,08)$ \\
Auxiliar de enfermagem & 2 & $(4,08)$ \\
Representante comercial & 1 & $(2,04)$ \\
Outro & 33 & $(67,35)$ \\
\hline Escolaridade & Analfabeto & 15 & $(30,00)$ \\
Fundamental & 19 & $(38,00)$ \\
Médio incompleto & 4 & $(8,00)$ \\
Médio completo & 8 & $(16,00)$ \\
Superior & 4 & $(8,00)$ \\
Médio completo ou superior & 12 & $(24,00)$ \\
Até médio incompleto & 38 & $(76,00)$ \\
\hline
\end{tabular}

Fonte: Dados da pesquisa.

Em relação ao perfil sociodemográfico (Tabela 1), pode-se inferir que há uma predominância de idosos com idade menor de 70 anos $(62,0 \%)$, sexo feminino $(66,0 \%)$ e raça branca $(58,0 \%)$. Ao estado civil, metade dos idosos eram casados $(50,0 \%)$ e $(32,65 \%)$ deles eram aposentados. Em relação a realização da atividade da laboral, $(67,35 \%)$ as realizavam, sendo a atividade que apareceu com maior frequência, era a condição de dona de casa $(28,57 \%)$. Quanto a escolaridade verificou-se que (30,0\%) não eram alfabetizados; $(38,0 \%)$ apresentavam ensino fundamental; $(8,0 \%)$ ensino médio incompleto; $(16,0 \%)$ ensino médio completo; $(24,0 \%)$ ensino superior completo e $(76,0 \%)$ ensino superior incompleto. 
Tabela 2: Comparação de médias da Escala de Barthel em relação ao risco de quedas a idosos. Vitória da Conquista/BA, 2017.

\begin{tabular}{lccc}
\hline & \multicolumn{2}{c}{ RISCO_QUEDA } & \\
\hline \multirow{2}{*}{ Variáveis } & Baixo & Alto & p-valor \\
\cline { 2 - 4 } Alimentação & Média & Média & 0,39 \\
Banho & 10,00 & 9,83 & 1,00 \\
Atividades & 5,00 & 5,00 & 1,00 \\
Vestir & 5,00 & 5,00 & 0,22 \\
Intestino & 10,00 & 9,66 & 0,80 \\
Urinário & 9,52 & 9,31 & 0,68 \\
Toillet & 9,29 & 9,48 & 1,00 \\
Transferência & 10,00 & 10,00 & 0,30 \\
Mobilidade & 14,76 & 14,31 & 0,76 \\
Escadas & 14,76 & 14,66 & 0,76 \\
Total & 9,76 & 9,66 & 0,29 \\
\hline
\end{tabular}

Fonte: Dados da pesquisa.

A tabela 2 acima, trás a comparação das médias da escala de Barthel, e podemos observar que não houve estatística se comparada ao escores de cada domínio relacionado ao risco de quedas e ao valor (p-valor) maior que $(0,05)$. O que nos mostra que em relação a aplicação desta escala, há uma grande dependência e riscos de incapacidade funcional entre os idosos estudados.

Tabela 3: Comparação de médias da Escala de Lawton quanto ao risco de quedas em idosos. Vitória da Conquista/BA, 2017.

\begin{tabular}{lccc}
\hline & \multicolumn{3}{c}{ RISCO_QUEDA } \\
Variáveis & 2,86 & Alto & p-valor \\
\cline { 2 - 4 } & Média & Média & 0,70 \\
Telefone & 2,86 & 2,86 & 0,30 \\
Transporte & 2,95 & 2,86 & 0,47 \\
Compras & 3,00 & 2,83 & 0,13
\end{tabular}




\begin{tabular}{lccc} 
Arrumar a casa & 2,95 & 2,79 & 0,28 \\
Trabalhos manuais & 2,86 & 2,93 & 0,70 \\
Lavar e passar & 2,86 & 2,86 & 0,94 \\
Tomar remédios & 2,90 & 2,93 & 0,74 \\
Finanças & 2,90 & 2,62 & 0,10 \\
Total & 26,24 & 25,48 & 0,24 \\
\hline
\end{tabular}

Fonte: Dados da pesquisa.

A avaliação da capacidade funcional foi realizada através da aplicação da escala de Lawnton (Tabela 3). Perante as variáveis, nenhum idoso da amostra estudada apresentou grau de dependência total. $O$ ( $p$-valor) foi maior do que $(0,05)$, o que indica que não houve diferença estatística em relação ao risco de quedas.

Tabela 4: Comparação de médias do questionário de Risco de Quedas (Falls of Risk). Vitória da Conquista/BA, 2017.

\begin{tabular}{lccc}
\hline \multicolumn{3}{c}{ RISCO_QUEDA } \\
\hline \multicolumn{1}{c}{ Variáveis } & Baixo & Alto & p-valor \\
\cline { 2 - 4 } & Média & Média & 0,004 \\
Quedas anteriores & 0,62 & 1,07 & 0,013 \\
Medicações & 0,67 & 1,28 & 0,016 \\
Déficit sensório & 0,52 & 0,90 & 0,275 \\
Estado mental & 1,74 & 1,55 & 0,498 \\
Marcha & 0,43 & 0,14 & $<0,001$ \\
Total & 1,38 & 3,41 & \\
\hline
\end{tabular}

Fonte: Dados da pesquisa.

A tabela 4, retrata a comparação das médias do questionário sobre Risco de Quedas (Falls Risk Score) por Dowton. Em relação as variáveis relacionadas a quedas anteriores (Média alta), a média encontrada foi de $(1,07)$. Se comparada a (Média baixa) de $(0,62)$, podese inferir que houve prevalência ao risco de quedas a idosos, pois já ocorreu um evento pósqueda. $\mathrm{O}$ medo de cair pode provocar então o ciclo vicioso podendo influenciar na redução da capacidade funcional e a maior suscetibilidade a sofrer novas quedas.

Quanto uso de medicações, ocorreu um aumento estatístico nas médias de $(1,28)$ com relação ao alto risco de quedas e $(0,62)$ em relação ao baixo risco de quedas. Nas médias do 
déficit sensorial com alto risco de quedas encontrou-se a média de $(0,90)$, e idosos que apresentam baixo risco de quedas $(0,52)$. Foi realizada a divisão das classes entre baixo e alto risco de quedas e comparado aos escores de cada variável com valor ( $\mathrm{p}$-valor) $<(0,05)$, para quedas anteriores a $(0,004)$, com uso de medicações anti-hipertensivas $(0,013)$, e com déficit sensório $(0,016)$. Nota-se pontuação total de $<(0,001)$, indicando médias estatísticas diferenciadas entre os indivíduos de baixo e alto risco de quedas. Constata-se assim fator relevante ao risco de quedas nos idosos no que se refere ao uso de medicações e déficit sensório.

Tabela 5: Comparação de médias quanto a chance de risco de quedas segundo ao sociodemográfico, MEEM, GDS, TUG e IMC dos idosos. Vitória da Conquista/BA, 2017.

Risco de queda

\begin{tabular}{|c|c|c|c|c|c|c|c|c|c|}
\hline & & & aixo & & Alto & & & & \\
\hline Variável & Classe & $\mathbf{N}$ & $(\%)$ & $\mathbf{N}$ & $(\%)$ & p-valor & OR & IC & $\mathrm{C}$ \\
\hline Idade & $<70$ & 13 & $(61,90)$ & 17 & $(58,62)$ & 0,53 & 1,15 & 0,36 & 3,62 \\
\hline & $>=70$ & 8 & $(38,10)$ & 12 & $(41,38)$ & & & & \\
\hline Sexo & Masculino & 11 & $(52,38)$ & 8 & $(27,59)$ & 0,07 & 2,89 & 0,89 & 9,41 \\
\hline & Feminino & 10 & $(47,62)$ & 21 & $(72,41)$ & & & & \\
\hline Cor & Branco & 10 & $(47,62)$ & 19 & $(65,52)$ & 0,17 & 0,48 & 0,15 & 1,51 \\
\hline & Não branco & 11 & $(52,38)$ & 10 & $(34,48)$ & & & & \\
\hline Profissão & Outro & 14 & $(70,00)$ & 19 & $(65,52)$ & 0,49 & 1,23 & 0,36 & 4,18 \\
\hline & Aposentado & 6 & $(30,00)$ & 10 & $(34,48)$ & & & & \\
\hline Escolaridade & Médio completo/sup. & 3 & $(14,29)$ & 9 & $(31,03)$ & 0,15 & 0,37 & 0,09 & 1,59 \\
\hline & $\begin{array}{l}\text { Até médio } \\
\text { incompleto }\end{array}$ & 18 & $(85,71)$ & 20 & $(68,97)$ & & & & \\
\hline Mini_Mental & Incapaz & 6 & $(31,58)$ & 13 & $(44,83)$ & 0,27 & 0,57 & 0,17 & 1,91 \\
\hline & Capaz & 13 & $(68,42)$ & 16 & $(55,17)$ & & & & \\
\hline GDS & Depressão & 4 & $(19,05)$ & 5 & $(17,24)$ & 0,58 & 1,13 & 0,26 & 4,84 \\
\hline & Sem depressão & 17 & $(80,95)$ & 24 & $(82,76)$ & & & & \\
\hline MOB_FLEX & Incapacitado & 0 & $(0,00)$ & 2 & $(6,90)$ & 0,33 & 1,78 & 1,39 & 2,28 \\
\hline & Capacitado & 21 & $(100,00)$ & & $(93,10)$ & & & & \\
\hline Confiante para teste & Sim & 21 & $(100,00)$ & 27 & $(93,10)$ & 0,33 & 0,56 & 0,44 & 0,72 \\
\hline & Não & 0 & $(0,00)$ & 2 & $(6,90)$ & & & & \\
\hline Catarata & Sim & 1 & $(4,76)$ & 4 & $(13,79)$ & 0,29 & 0,31 & 0,03 & 3,02 \\
\hline & Não & 20 & $(95,24)$ & 25 & $(86,21)$ & & & & \\
\hline IMC & $<25$ & 6 & $(37,50)$ & 13 & $(48,15)$ & 0,36 & 0,65 & 0,18 & 2,28 \\
\hline
\end{tabular}




\section{Fonte: Dados da pesquisa.}

Em relação a Tabela 5, verificam-se duas análises importantes: o teste de associação e a chance de risco (odds ratio). O teste de associação tem como resultado o (p-valor) na tabela. Para esse, ( $\mathrm{p}$-valor) menor do que $(0,05)$, significa associação entre as variáveis linha $\mathrm{x}$ coluna. Já relacionado as análises de chance de risco (do inglês odss ratio - OR), os resultados são do próprio OR e do seu intervalo de confiança (IC). Foi realizado também o teste exato de Fisher (que tem o mesmo significado do teste de qui-quadrado). Estes testes de associação avaliam de certa forma a relação entre as variáveis qualitativas, onde em nenhuma delas houveram associações quanto ao risco de quedas.

Realizada a diferenciação entre a proporção das freqüências e comparada as médias relacionadas ao sociodemográfico; tem-se entre o baixo e o alto risco de quedas nas variáveis: Idade: < 70 anos $(61,90 \%)$ e $(58,62 \%)$. Sexo feminino $(47,62 \%)$ e $(72,41 \%)$; Cor: Branca $(47,62 \%)$ e $(72,41 \%)$. Profissão: Outro $(70,00 \%)$ e $(65,52 \%)$. Escolaridade: Ensino médio incompleto $(85,71 \%)$ e $(68,97 \%)$. O Mini Mental: Capazes $(68,42 \%)$ e $(55,17 \%)$. Escala de depressão (GDS): Sem depressão (80,95\%) e (82,96\%). Mobilidade e flexibilidade: Capacitado (100,00\%); e o IMC: >= $25(62,50 \%)$ e (51,85\%). Entre os idosos não foram encontradas diferenças estatísticas nas classificações entre baixo e alto risco de quedas.

Observando todas as variáveis da tabela 5 , com relação ao teste que foi realizado para verificar a chance do risco de quedas, em nenhuma delas o (p-valor) foi menor que $(0,05)$. Pelos resultados das variáveis não foi possível mostrar fator significante para o risco de quedas, o que traduz a coerência das tabelas anteriores, estando evidenciado que os idosos estudados são capazes e independentes para realizar a qualquer tarefa diária, não apresentando relevância em relação ao risco de quedas, exceto relacionado ao questionário Risco de Quedas (Falls Risk Downton), onde destacou quedas nessa população de idosos no valor (p-valor) de $<(0,001)$ tendo como as causas mais relevantes foram: as associadas a quedas anteriores, uso de medicações e déficit sensório.

\section{Discussão}


Quanto ao perfil sociodemográfico e econômico foi visto que $(62,0 \%)$ representaram os idosos do sexo feminino, corroborando ao estudo realizado por Nascimento e Tavares (2016) em Uberaba-MG, que destacou a ocorrência de quedas ao sexo feminino. Tal relação pode se justificar pela constatação quanto a presença predominante (em número) de idosos do sexo feminino em atendimento nas unidades básicas de saúde e comprovado pela amostra de idosos entre feminino e masculino nesta pesquisa.

Entre os idosos participantes deste estudo, (60,0\%) tem menos de 70 anos de idade, o que corrobora com o estudo de Siqueira et al. (2007), tendo (38,5\%) entre 65 e 70 anos. Tal fato denota que quanto maior a idade, maior é a probabilidade em apresentar níveis de incapacidade aos quais associados a fatores tais como depressão, uso de medicamentos, fatores fisiológicos, declínio cognitivo, perda do equilíbrio, fraqueza muscular e distúrbios da marcha entre outros fatores associados que intensificam a relevante importância ao estudo sobre o tema.

Ao estado civil, (50,0\%) casados, divergindo do estudo desenvolvido por Rosa et al. (2015) onde, em relação perfil epidemiológico dos que sofreram algum tipo de queda eram viúvos. Tal distinção justificada pela baixa incidência de divórcio nesta população.

Quanto a raça, foi evidenciado que $(58,0 \%)$ brancos, discordando do estudo realizado por Freitas et al. (2015) onde, feito inquérito dos serviços de Urgência do Sistema Único de Saúde com idosos vítimas de quedas e acidentes de trânsito haviam mais negros que brancos. O Brasil é um país que possui grande confluência de pessoas de diversas origens porém o acesso básico regular ainda não se evidencia de forma igualitária, e causa diferenças comparáveis entre as unidades de saúde de atendimento básico a urgência e emergência.

Constatou-se que $(38,0 \%)$ possuíam ensino fundamental completo, confrontando com Neto et al. (2017), em que (35,6\%) eram analfabetos. Visto que pessoas com baixa escolaridade podem apresentar maior déficit cognitivo, problemas psicológicos e/ou outros distúrbios que pode influenciar nas quedas; na amostra estudada os idosos apresentaram regular nível de formação, o que evidencia em sua condição vida social.

Reiterando a variável "mora com quantas pessoas" os idosos residiam com $(3,22)$ pessoas em média, corroborando ao o estudo de Cruz et al. (2012), em que (90,0\%) dos idosos pesquisados residiam com outras pessoas. A julgar que esse público necessita de cuidados especiais, um responsável faz-se necessário ao auxílio das as atividades da vida diária, ainda que entre os pesquisados atendidos foi constatada a presença de familiar e/ou cuidador. 
No presente estudo, por meio dos dados encontrados através da Escala de Lawton, $(68,0 \%)$ dos idosos eram independentes.; discordando de Gomercindo e Garcez (2012), onde $(100,0 \%)$ eram dependentes. Identificou-se maior relevância a dependência nos escores: Banho, vestuário, higiene e as transferências segundo AIVDS. De acordo com a Escala de Lawton essa dependência se deu significativamente entre os idosos por conta da idade avançada e a associação a sequelas a alguma patologia, exemplo o AVE (Acidente Vascular Encefálico).

Estudo realizado por França et al. (2011) sobre as atividades da vida diária entre os idosos adscritos às Unidades Básicas de Saúde, área urbana de Campina Grande-PB; investigou a condição de saúde e estimou a incapacidade funcional leve com $(34,0 \%)$ e grave (8,0\%). Idosos entre 69 e 70 anos (14,0\%), e os demais com idade avançada e baixa escolaridade. Este resultado contrasta com o resultado obtido, não havendo relação significante a incapacidade funcional a ser considerado fator de quedas, podendo ser compreendida quando associada a idade, que neste estudo que foi de $(60,0 \%)$, menor que 70 anos e o nível escolar satisfatório.

Além das questões sociodemográficas e do Índice de Barthel, foram avaliados aspectos precedentes por meio de outros instrumentos como a Antropometria, verificando que a prevalência de quedas nos últimos 12 meses foi de (15,8\%), destacando-se em idosos do sexo feminino; corroborando com o que foi encontrado no estudo de Silva et al. (2013), Teresina/PI, onde correlacionou o risco de quedas a autonomia funcional dos idosos, e a maior parte dos idosos eram do sexo feminino, e cursaram com maior prevalência a quedas.

Em saúde mental, a diferença entre os sexos foi evidenciada quanto ao declínio cognitivo, onde, idosos do sexo feminino apresentaram perda cognitiva maior em relação ao masculino; desta forma, atestando ao estudo sobre o perfil cognitivo de mulheres institucionalizadas de Machado et al. (2011) em Brasília-DF; sendo também as mulheres as que apresentaram declínio cognitivo. Este aspecto pode ser justificado relacionado ao nível de escolaridade, situação familiar e conjugal atribuída pela condição cultural e histórica da população brasileira.

Nos resultados encontrados no TUG relacionados à mobilidade e flexibilidade, foi constatada a capacitação para tal função; concordando ao estudo realizado por Ruzene e Navega (2014), onde mostrou resultados sobre a mobilidade e flexibilidade em idosos praticantes e não praticantes de exercício físico, sendo os que praticam exercício físico, seja 
ele aeróbio ou resistido, apresentam melhor mobilidade e melhor flexibilidade em relação ao que não praticam. Observado ainda em relação ao TUG, no que verifica a relação de confiança do idoso para realizar o teste, os idosos apresentaram-se aptos; corroborando como estudo de Martinez et al. (2016) onde menos de um terço tiveram dificuldade apenas na terceira mensuração. Os idosos avaliados em ambos os estudos apresentaram mobilidade e flexibilidade satisfatória, sendo então possível realizar o teste até a sua capacidade e da mesma forma comparada aos idosos da amostra. Neste mesmo instrumento de coleta foram avaliados os idosos submetidos a cirurgia de correção da catarata, ao qual a considerar que quanto menor o campo de visão, maior é a necessidade de atenção a locomoção, podendo em sua falta ocorrer o aumento do risco de quedas.

No instrumento para avaliação do risco de quedas, idosos relataram não terem caído anteriormente; contrariando o estudo de Campos et al. (2017) onde (85,0\%) dos idosos possuem conhecimentos sobre queda através da própria experiência de vida. O uso de medicações e/ou automedicação no público pesquisado não foi fator relevante, a considerar que este público possui acompanhamento, o que também não evidencia o uso de psicoativos. O resultado foi diferente a exemplo de Araújo e Galato (2012) onde amostras com 135 idosos $(70,8 \%)$ faziam uso de medicamentos, diferenciando-se assim quanto a quedas, cuja maioria que faz uso dos benzodiazepínicos relataram quedas anteriores e redução da cognição.

A maioria dos idosos pesquisados não possuem catarata $(95,2 \%)$, considerando também os submetidos a correção. A área de visão é um parâmetro importante na avaliação quanto ao risco de quedas e depressão. Sua deficiência a atividades corriqueiras no próprio domicílio sem adaptações adequadas são agravantes ao risco.

Quanto ao déficit sensorial, majoritariamente, os participantes apresentavam visão prejudicada em sua condição de longevidade; discordando do estudo realizado por Smith et al. (2017), pois os idosos de sua pesquisa não apresentavam alterações visuais. Estas relações podem ser explicadas considerando-se que em regiões com clima frio e seco existam preponderância a problemas de visão tais como glaucoma e catarata, podendo comprometer a qualidade de vida, além do aumentar ao risco de quedas e outros acidentes.

Em relação ao estado mental, os idosos apresentaram estado cognitivo preservado, se distanciando ao estudo realizado por Machado et al., (2011), no qual o índice de idosos com declínio cognitivo foi alto. Para esta variável que depende de fatores como escolaridade e estilo de vida, quanto mais ativo for o idoso potencialmente poderá ser seu declínio físico, 
entretanto fatores extrínsecos podem contribuir parar tal estado mental como o uso de medicamentos por tempo prolongado. A marcha dos participantes na sua maioria, apresentouse normal, discordando do estudo de Gomes et al. (2016), onde os idosos apresentaram alterações nos parâmetros espaço-temporais da marcha, como velocidade, comprimento da passada, cadência e tempos de fases da marcha durante o desempenho de dupla tarefa.

Por meio do questionário de risco de quedas evidenciou-se alto risco. O indivíduo passa por diversas fases as quais estão expostos às alterações fisiológicas, o aparecimento de doenças que contribuem para sua debilidade, e os idosos são mais acometidos por estas alterações que geram fraqueza muscular, declínio cognitivo e deficiência nutricional influenciando gradativamente ao risco de quedas. Esta evidência corrobora com o estudo de Pinho et al. (2012) avaliou também o risco de quedas dos idosos em Unidade Básica de Saúde, e o que diferencia sua pesquisa deste presente estudo, foi apenas a amostra, constituída de 150 idosos pesquisados.

Ao índice de depressão, o estudo revelou que $(80,95 \%)$ dos idosos não apresentaram nenhum tipo de depressão, seja ela leve, moderada ou grave. Resultado que exerce uma importante função na redução do risco de quedas. Essa patologia gera fadiga, perda de massa muscular e redução da cognição aumentando o risco de quedas, o que não se aplica aos idosos do presente estudo. Sobre mobilidade e flexibilidade, foi satisfatório o item locomoção e equilíbrio, o que indica redução significativa a quedas.

Um estudo realizado por Soares et al. (2013), observou que (41,0\%) apresentaram algum tipo depressão em idosos assistidos nas Unidades Básicas de Saúde pesquisadas. No estudo realizado por Silva et al., (2012), dos idosos (64,3\%) exprimiam quadro depressivo e $(58,1 \%)$ eram do sexo feminino. Neste estudo não houve significância em relação aos estudos citados, tanto em relação a quantidade e/ou gênero; embora a mulher em idade avançada tende ser mais ansiosa e predisposta a frustrações, que sem acompanhamento pode desencadear outros problemas funcionais.

Em relação ao IMC, a maioria dos idosos apresentaram o Índice de Massa Corpórea $(\geq 25)$, indicando um sobrepeso e tornando um fator de risco alto para quedas, uma vez que também desencadeia outras alterações e favorece o desenvolvimento de patologias. A dificuldade a locomoção foi visto que na classificação para alto risco de quedas a única variável que apresentou média acima do geral foi 51,85\%, para idosos com sobrepeso. Estes valores associados e utilizados com o padrão de referência a idade e gênero dos idosos e 
relacionado as diferenças entre tamanho e estrutura muscular, corroboram então com o estudo de Silva et al. (2015), que foi semelhante a este presente estudo e verificou a prevalência de sobrepeso com 43,50\% dos idosos atendidos em ambulatório de nutrição em Niterói-RJ.

Durante o processo de coleta dos dados, houveram casos em foi necessário o auxílio do familiar que estava presente no momento, para complementar as respostas ao idoso que apresentou dificuldade a assimilação das questões propostas. O número quantitativo da amostra dos idosos pesquisados relaciona-se aos critérios de inclusão, estando para tal, os resultados significativos para a população descrita. Tais dados concedem associações sem causas inferenciais. Considera-se ainda, a falta de estudos e pesquisas recentes relacionadas ao público idoso.

\section{Considerações Finais}

Os idosos evidenciam fatores intrínsecos a sua condição de vida e se relacionam a parâmetros sociodemográficos, antropométricos, econômicos e psicossociais. Suas influências condizem a cerca da idade relacionada ao risco de quedas, longevidade e qualidade de vida. Estas relações foram estudadas e descrevem idosos em condição de atendimento regular na Atenção Primária à Saúde.

Os idosos apresentaram em média idade inferior a 70 anos com predominância do sexo feminino. Todos aposentados e renda compatível a sua condição social. Em parte casados, possuem filhos e realizam atividade laboral profissional e doméstica. Poucos não alfabetizados e proeminência a idosos com nível superior incompleto. O risco de quedas referenciado foi atribuído a quedas anteriores, uso de medicações e déficit sensório e o uso de medicações foi fator relevante e representativo, mesmo sem constar automedicação declarada.

Mediante os resultados encontrados, as quedas entre os idosos nestas condições expostas estão assim identificadas e relacionadas ao uso de medicamentos, quedas anteriores, cognição, flexibilidade e mobilidade. Os idosos estudados apresentaram no geral, resultados satisfatórios, sendo capazes, independentes e com aptidão a realização de tarefas comuns a idade. Desta forma constata-se que o aprimoramento, acompanhamento e constantes estudos sobre idosos são necessários, frente a demanda constante em atendimento humanizado e 
especializado, e que respondam as características distintas desta população em suas nuances relacionadas a longevidade e as atuais condições de vida.

\section{Referências}

ARAÚJO, P.L.; GALATA, D. Risco de Fragilização e Uso De Medicamentos em Idosos Residentes em Uma Localidade do Sul de Santa Catarina. Revista Brasileira de Geriatria e Gerontologia, Rio de Janeiro; 15(1):119-126, 2012.

CAMPOS, Karis de. Capacitação de idosos na prevenção de quedas domiciliares utilizando tecnologias da informação e comunicação. Revista de Atenção à Saúde RAS, 2017.

CRUZ, D.T.; et al. Prevalência de Quedas e Fatores Associados em Idosos. Revista de Saúde Pública; 46(1):138-46, 2012.

FALSARELLA, G. R.; GASPAROTTO, L. P. R.; COIMBRA, A. M. V. Quedas: conceitos, frequências e aplicações à assistência ao idoso. Revisão da literatura. Rev. bras. geriatr. gerontol. Vol.17, n.4, pp.897-910, 2014.

FRANÇA, I.S.X.; et al. Condições Referidas de Saúde e Grau de Incapacidade Funcional em Idosos. Revista Rene; abr/jun; 12(2):333-41, 2011.

FREITAS, M.G.; et al. Idosos Atendidos em Serviços de Urgência no Brasil: Um Estudo para Vítimas de Quedas e de Acidentes de Trânsito. Revista Ciência \& Saúde Coletiva, 20(3):701-712, 2015.

FOLSTEIN, R.R.G.; FOLSTEIN,M.; MCHUGH,P. R. R. Reduced caloric intake following small bowel by-pass surgery: a systematic study of possible causes. Psychological Medicine, v. 9, n. 01, 1975.

GIL, A.C. Métodos e Técnicas de Pesquisa Social. 6 ed. São Paulo: Atlas, 2017.

GOMERCINDO, M.C.H.; GARCEZ, E.M.S. Avaliação da Capacidade Funcional de Idosos de Uma Comunidade do Município de Porto União Em Santa Catarina. Revista de Saúde Pública de Santa Catarina; v. 5, n. 2, p. 30- 45, maio/ago. 2012.

GOMES, G.C.; et al. Desempenho de Idosos na Marcha com Dupla Tarefa: Uma Revisão dos Instrumentos e Parâmetros Cinemáticos Utilizados Para Análise. Revista Brasileira de Geriatria e Gerontologia, Rio de Janeiro; 19(1):165-182, 2016.

MACHADO, J.C.; et al. Declínio Cognitivo de Idosos e Sua Associação Com Fatores Epidemiológicos em Viçosa, Minas Gerais. Revista Brasileira de Geriatria e Gerontologia; Rio de Janeiro; 14(1):109-121, 2011.

MARTINEZ, B.P.; et al. Segurança e Reprodutibilidade do Teste Timed Up And Go em Idosos Hospitalizados. Revista Brasileira de Medicina do Esporte - Vol. 22, No 5 - Set/Out, 2016. 
NASCIMENTO, J.S.; TAVARES, D.M.S. Prevalência e Fatores Associados a Quedas em Idosos. Texto Contexto Enfermagem; 25(2):e0360015, 2016.

NETO, A.H.A.; et al. Quedas em Idosos Institucionalizados: Riscos, Consequências e Antecedentes. Revista Brasileira de Enfermagem; jul-ago;70(4):752-8, 2017.

PAULA, Ludmila Schettino Ribeiro de. Modelo preditivo do risco de quedas para idosos residentes em comunidade. Dissertação (Programa de Pós-Graduação em Enfermagem e Saúde), Universidade Estadual do Sudoeste da Bahia, Jequié, 2015.

PINHO, T.A.M de.; et al. Avaliação do Risco de Quedas em idosos atendidos em Unidade Básica de Saúde. Revista da Escola de Enfermagem da USP; vol.46 n.2 São Paulo, 2012.

ROSA, T.S.M.; et al. Perfil Epidemiológico de Idosos que Foram a Óbito por Queda no Rio Grande do Sul. Revista Brasileira de Geriatria e Gerontologia, Rio de Janeiro; 18(1):59-69, 2015.

SANTOS, Thayane Dias do. O ambiente do cuidado e a segurança do paciente hospitalizado: contribuições para a enfermagem. Dissertação ( Programa de Pós-Graduação em Ciências do Cuidado em Saúde Curso de Mestrado em Ciências do Cuidado em Saúde), Escola de Enfermagem Aurora de Afonso Costa, UFF, Niterói, 2017.

SILVA, E.R.; et al. Prevalência e fatores associados à depressão entre idosos institucionalizados: subsídio ao cuidado de enfermagem. Revista da Escola de Enfermagem da USP; 46(6):1387-93, 2012.

SILVA, A. S. da. C. S.; et al. Avaliação Antropométrica de Idosos Atendidos no Ambulatório de Nutrição do Centro de Referência em Assistência à Saúde do Idoso da Universidade Federal Fluminense, no Município de Niterói-RJ. Revista Demetra: Alimentação, Nutrição e Saúde, Rio de Janeiro; v.10, 2015.

SILVA, J.M.N.; et al. Correlação Entre o Risco de Queda e Autonomia Funcional em Idosos Institucionalizados. Revista Brasileira de Geriatria e Gerontologia, Rio de Janeiro; 16(2):337-346, 2013.

SIQUEIRA, F.V.; et al. Prevalência de Quedas em Idosos e Fatores Associados. Revista de Saúde Pública; 41(5):749-56, 2007.

SMITH, A.A.; et al. Avaliação do Risco de Quedas em Idosos Residentes em Domicílio. Revista Latino-Americana de Enfermagem; 25:e2754, 2017.

SOARES, P. F. C.; et al. Depressão em Idosos Assistidos nas Unidades Básicas de Saúde. Revista de Enfermagem UFEP on line, Recife; 7(9):5453-9, 2013.

RUZENE, J. R. S., NAVEGA, M. T., Avaliação do equilíbrio, mobilidade e flexibilidade em idosas ativas e sedentárias. Revista Brasileira de Geriatria e Gerontologia, 2014.

Como citar este artigo (Formato ABNT): 
LEMOS, Gabriela F.; FERREIRA, Jualiana B.; SANTOS, Kleyton T.; REIS, Luciana A. dos R; MORAIS, Karla C. S. de. Fatores Associados a Quedas em Idosos de uma Unidade Básica de Saúde. Id on Line Revista Multidisciplinar e de Psicologia, 2017, vol.11, n.38, p.150-165. ISSN: 1981-1179.

Recebido: 23.10.2017

Aceito: 24.10 .2017 